\title{
malestar emocional, ansiedad y depresión en pacientes oncológicos colombianos y su relación con la competencia percibida
}

\section{Distress, fnxiety and Depression in Colombian Oncology Patients and their Relation with Perceived Competence \\ mal-estar emocional, ansiedade e depressão em pacientes oncológicos colombianos e sua relação com a concorrência percebida}

\author{
Ana Isabel Moreno Acosta*,Alicia Krikorian*, Carolina Palacio* \\ Universidad Pontificia Bolivariana, Medellín, Colombia
}

Doi: dx.doi.org/10.12804/apl33.03.2015.10

\section{Resumen}

Objetivos: Determinar los índices de malestar emocional, ansiedad y depresión y examinar su relación con la competencia percibida $(\mathrm{CP})$ en pacientes con cáncer. Método: Se recogieron datos sociodemográficos y clínicos de 42 pacientes que consultaron un servicio de psicología de una institución oncológica. Se empleó la Escala de Ansiedad y Depresión Hospitalaria (HADS) y la Escala de Competencia Percibida (ECP). Se obtuvieron estadísticos descriptivos, comparativos y correlacionales. Resultados: Edad promedio $49 \pm 10.6$ años (rango 22-69), 83\% de sexo femenino, diagnóstico más frecuente: cáncer de mama (26.2\%). El 61.9\% se encontraba en estadio IV, y más del 50\%, en tratamiento paliativo. Las puntuaciones en malestar emocional, ansiedad y depresión fueron bajas-medias, y las de CP fueron moderadas. Se observó una relación inversa y significativa entre el malestar emocional y la CP $(p<$ $.01)$. Sin embargo, la correlación entre ansiedad o depresión y CP no fue significativa. Conclusiones: Las creencias de control generales mantienen una relación estrecha con el estado emocional. El investigar sobre la CP tiene ventajas importantes, ya que las creencias generales de control pueden favorecer la adaptación en el proceso de enfermedad.

Palabras clave: malestar emocional; competencia percibida; cáncer; ansiedad; depresión.

\section{fbstract}

Objective: To determine the levels of distress, anxiety and depression and their relation with perceived competence (PC) in cancer patients. Methods: Sociodemo-

* Ana Isabel Moreno Acosta, Alicia Krikorian, miembro del Grupo de Dolor y Cuidado Paliativo, Escuela de Ciencias de la Salud, Universidad Pontificia Bolivariana; Carolina Palacio, Instituto de Cancerología, Clínica Las Américas, y Miembro del Grupo de Dolor y Cuidado Paliativo, Escuela de Ciencias de la Salud, Universidad Pontificia Bolivariana.

La correspondencia relacionada con este artículo debe ser enviada a Alicia Krikorian, Escuela de Ciencias de la Salud, Oficina de Posgrados, calle 78B \# 72A-109, Universidad Pontificia Bolivariana, Medellín, Colombia. Correo electrónico: aliciakriko@, gmail.com

Cómo citar este artículo: Moreno, A. I., Krikorian, A. \& Palacio, C. (2015). Malestar emocional, ansiedad y depresión en pacientes oncológicos colombianos y su relación con la competencia percibida. Avances en Psicología Latinoamericana, 33(3), 517-529. doi: dx.doi.org/10.12804/ap133.03.2015.10 
graphic and clinical data of 42 patients attending the psychology service of an oncology institution were collected. Also, the Hospital Anxiety and Depression Scale (HADS) and the Perceived Competence Scale (PCS) were used. Descriptive, comparative and correlational statistics were obtained. Results: Mean age $49 \pm 10.6$ years (rank 22-69), 83\% female, the most frequent diagnosis was breast cancer $(26.2 \%), 61.9 \%$ were in stage IV and more than half in palliative treatment. Scores of distress, anxiety and depression were medium-low and were moderate for PC. An inverse and significant relation between distress and PC was found $(p<.01)$. Correlations between anxiety, depression and PC were non-significant. Conclusions: General control beliefs such as PC are closely related to the emotional state of patients. Research on PC is of importance given that general control beliefs may benefit adjustment along the illness trajectory.

Keywords: distress; perceived competency; cancer; anxiety; depression.

\section{Resumo}

Objetivo: determinar os níveis de mal-estar emocional, ansiedade e depressão e examinar a sua relação com a Concorrência Percebida (CP) em pacientes com cáncer. Método: recolheram-se dados sociodemográficos e clínicos de 42 pacientes que consultaram a um serviço de psicologia de uma instituição oncológica. Empregou-se a Escala de Ansiedade e Depressão Hospitalar (HADS) e a Escala de Concorrência Percebida (ECP). Obtiveram-se estatísticos descritivos, comparativos e correlacionais. Resultados: Idade média $49 \pm 10.6$ anos (rango 22-69), 83\% de gênero feminino, diagnóstico mais frequente: cáncer de mama (26.2\%). O 61.9\% encontrava-se em estádio IV e mais do $50 \%$ em tratamento paliativo. As pontuações em mal-estar emocional, ansiedade e depressão foram baixas-meias e as de CP foram moderadas. Observou-se uma relação inversa e significativa entre o mal-estar emocional e a CP $(\mathrm{p}<0.01)$. No entanto, a correlação entre ansiedade ou depressão e CP não foi significativa. Conclusões: as crenças de controle gerais mantêm uma relação estreita com o es- tado emocional. A pesquisa sobre a $\mathrm{CP}$ tem vantagens importantes devido a que as crenças gerais de controle podem favorecer a adaptação no processo de doença. Palavras-chave: mal-estar emocional; concorrência percebida; cáncer; ansiedade; depressão.

Según la Organización Mundial de la Salud (2008), el cáncer es una de las principales causas de muerte en el mundo. En 2012 hubo aproximadamente 14 millones de casos nuevos y se estima que los fallecimientos incrementen de 8.5 a 13 millones en las próximas dos décadas (International Agency for Cancer Research, 2014). El 60\% de los casos se presenta en países con ingresos bajos o medios. En Colombia, las estimaciones de incidencia en el periodo 2000-2006 indican cerca de 70887 casos nuevos de cáncer por año (Instituto Nacional de Cancerología, 2010); mientras que en 2010 murieron alrededor de 33500 personas, con una tasa de mortalidad cercana a 73 por cada 100000 habitantes, que representa el 16.9\% del total de muertes para el país. Estas elevadas tasas de incidencia y mortalidad reflejan una grave situación que se podría explicar por la baja eficacia en los tratamientos y la tendencia a hacer diagnósticos en estadios tardíos de la enfermedad (Ministerio de Salud y Protección Social \& Instituto Nacional de Cancerología, 2012).

El cáncer puede traer consigo un impacto en las dimensiones física, psicológica, social y espiritual (Rebollo \& Aguilar, 2006), y su diagnóstico favorece el desarrollo de problemas para el paciente, la familia o la comunidad, en cualquier fase de su trayectoria (Bultz \& Carlson, 2006; Bultz \& Johansen, 2011). Una vez la enfermedad es diagnosticada, ingresa a la cotidianidad de la persona y de su entorno y afecta la seguridad y la confianza, puesto que se introducen cambios para los cuales el enfermo no se había preparado. Con el transcurrir del tiempo, la persona inicia un proceso de aceptación lenta y progresiva a la idea de la existencia de la enfermedad en su vida, así como de adapta- 
ción a los cambios mencionados (Krikorian, 2008; Hernández et al., 2012) y a las pérdidas que esta conlleva, incluidos la reorganización del medio familiar, emocional y la elaboración de duelos de aquellas situaciones a las que diariamente se ve obligado a renunciar (Bultz \& Carlson, 2006; Rebollo \& Aguilar, 2006).

El proceso de adaptación se puede acompañar de reacciones emocionales diversas: impotencia, irritabilidad, desesperanza, pérdida de control, ansiedad, estrés, tristeza, depresión, miedo, entre otros. Numerosos autores han tratado el tema del malestar emocional, la ansiedad y la depresión en enfermos con diferentes diagnósticos y estadios de cáncer (Carlson et al., 2004; Delgado-Guay, Parsons, Li, Palmer \& Bruera, 2009; Mystakidou et al., 2010; Ornelas-Mejorada, Tufiño \& SánchezSosa, 2011; Finck y Forero, 2011; Rosenstein, 2011; Mystakidou et al., 2012). De acuerdo con Costa y Ballester (2010), parece haber una mayor prevalencia de síntomas ansiosos que depresivos en pacientes oncológicos hospitalizados, los cuales tienden a disminuir en la trayectoria de enfermedad; mientras que los síntomas depresivos tienden a mantenerse o, incluso, a incrementar en el postratamiento o al avanzar la enfermedad.

En 2010, Ornelas-Mejorada et al., en un estudio realizado en mujeres mexicanas con cáncer de mama en tratamiento con radioterapia, encontraron una prevalencia de ansiedad y depresión del 27 y $28 \%$, respectivamente; las pacientes que se encontraban deprimidas presentaron más efectos secundarios que las que las que no lo estaban. Por su parte, Delgado-Guay et al. (2009) realizaron un estudio para determinar la relación entre la frecuencia y la intensidad de los síntomas físicos de pacientes con cáncer y sus expresiones de ansiedad y depresión. De un total de 216 pacientes, el 37\% presentó un estado de ánimo deprimido, y el 44\%, uno ansioso. Carlson et al. (2004) concluyeron en su trabajo que el malestar emocional es muy común en pacientes con cáncer a lo largo del diagnóstico y la trayectoria de la enfermedad; de hecho, los trastornos en la adaptación pueden ocurrir en el $35-50 \%$ de los enfermos (Kelly, McClement \& Chochinov, 2006; Mitchell, Karr, Coggan \& Herdman, 2008).

El logro de la adaptación a dichos cambios emocionales resultantes de la enfermedad requiere el empleo de una serie de esfuerzos de afrontamiento, en los cuales se ha visto que influyen factores cognitivos, como el grado de control percibido y la autoeficacia (Fernández \& Edo, 1994; Rebollo \& Aguilar, 2006; Rueda, Pérez-García \& Bermúdez, 2003; Rueda \& Pérez-García, 2004). El control percibido o sensación de control se refiere a la expectativa de participar en las decisiones para obtener consecuencias deseables y a la sensación de competencia personal en una situación determinada; en otras palabras, dicha sensación de control se logra cuando un sujeto considera que un agente, a través de unos medios, puede lograr unos resultados (Bárez, Blasco \& Fernández, 2003). La autoeficacia, por su parte, se refiere a los juicios o creencias personales acerca de las habilidades individuales necesarias para el logro de los resultados esperados (Bandura, 1986). Por tanto, la autoeficacia va a requerir la percepción de control individual en el proceso.

Diferentes estudios han indicado que la autoeficacia sirve como amortiguador del estrés psicológico derivado de los problemas y síntomas asociados al cáncer (Cunningham, Lockwood \& Cunningham, 1991; Hirai et al., 2002; Porter, Keefe, Garst, McBride \& Baucom, 2008). Por su parte, el control percibido o la sensación de control parece ser un ingrediente común relevante en los procesos terapéuticos y que favorecen el ajuste a la enfermedad, lo cual influye en el bienestar y la calidad de vida, al tiempo que disminuye el impacto emocional y la incertidumbre frente a la enfermedad (Bárez et al., 2003).

Adicional a los conceptos de control percibido y autoeficacia, se encuentra el de competencia percibida (CP), estrechamente relacionado y, de hecho, integra el control percibido y la autoeficacia (Bárez 
et al., 2003). Mientras la autoeficacia implica una mayor especificidad y hace referencia a recursos de acción específicos relacionados con una meta concreta, la $\mathrm{CP}$ se refiere a una creencia general y constituye un buen predictor de resultados en situaciones desconocidas, ambiguas o inciertas (Limonero, 1996; Limonero, Tomás-Sábado, Fernández-Castro, Cabellas \& Gómez, 2010; Fernández et al., 2012), como las que se presentan en la situación oncológica. En este sentido, la CP puede facilitar la evaluación de las situaciones adversas en términos de reto (e.g. la enfermedad), lo que favorece el empleo de estrategias activas de afrontamiento que impactan positivamente en la adaptación y el bienestar emocional (Rueda et al., 2003; Rueda \& Pérez-García, 2004; Hernández et al., 2012).

Sin embargo, son escasos los estudios que abordan la relación entre la CP y el cáncer, pues es una variable más estudiada en poblaciones con otro tipo de patologías. Por ejemplo, Martín et al. (2001) encontraron en su estudio que un locus de control externo se asociaba significativamente con ansiedad y depresión en pacientes con fibromialgia; mientras que la autoeficacia y la CP se relacionaban de forma cercana con la percepción de salud por lo que la CP constituye el mejor predictor del estado emocional. Por otro lado, Abraído-Lanza (1997), en un estudio llevado a cabo en mujeres latinas con artritis, encontró que la satisfacción con el rol y la sensación de CP mediaban el vínculo entre los estresores relacionados con la enfermedad y el ajuste psicológico. Por su parte, en 2009, Williams, Niemiec, Patrick, Ryan y Deci demostraron que una intervención orientada a desarrollar la autonomía y la autorregulación facilitaba la abstinencia al tabaco a largo plazo, resultado que se encontraba mediado por el incremento en la CP. Otros estudios ponen de manifiesto la relación entre la CP y la calidad de vida (Smith, Kielhofner \& Watts, 1986; Aubin, Hachey \& Mercier, 1999).

Teniendo en cuenta la escasez de estudios que aborden la relación entre malestar emocional y $\mathrm{CP}$ en pacientes con cáncer, así como los estudios en otras poblaciones que demuestran el impacto de esta última variable en el estado emocional, la calidad de vida y la adaptación a la enfermedad, se planteó la presente investigación, cuyo objetivo fue determinar los índices de malestar emocional, ansiedad y depresión y su relación con la CP en un grupo de pacientes con cáncer de la ciudad de Medellín (Colombia), que consultaron por primera vez el servicio de psicología de una institución oncológica.

\section{Metodología}

\section{Tipo de estudio y participantes}

Se realizó un estudio descriptivo, con diseño transversal. Participaron pacientes atendidos por primera vez en el servicio de psicología de una institución oncológica de la ciudad de Medellín, tanto en el servicio de hospitalización como en la consulta psicológica ambulatoria. La muestra total estuvo constituida por 42 adultos enfermos de cáncer, mayores de 18 años de edad, que se encontraban en diferentes estadios de la enfermedad y en diferentes etapas del tratamiento. Se excluyeron aquellos pacientes con problemas del sistema nervioso central que afectaran funciones cognitivas o en delírium, de acuerdo con lo consignado en la historia clínica, y pacientes que, por la gravedad de su condición médica o psicológica al momento de la entrevista, no estuvieran en capacidad de responder los cuestionarios.

\section{Procedimiento}

El estudio fue aprobado por el Comité de Ética institucional. Los participantes se seleccionaron de manera intencional, empleando un muestreo por conveniencia, a partir de los criterios de inclusión descritos. Se invitaron a participar en el estudio a todos los pacientes atendidos por primera vez por el servicio de psicología tanto ambulatoria como en hospitalización durante el periodo junio-agosto de 
2013 y que cumplían con los criterios de inclusión/ exclusión. Una vez se les explicó en qué consistía y se firmó un consentimiento informado, se citaron después de la consulta psicológica para recoger los datos sociodemográficos requeridos y aplicar los instrumentos de evaluación. La aplicación de los cuestionarios se hizo de forma autoaplicada y con el acompañamiento de una investigadora diferente a la psicóloga tratante. La investigadora explicó a cada paciente en qué consistía el cuestionario y su formato de aplicación. Así mismo, estuvo disponible para responder a las inquietudes que pudieran surgir durante el proceso.

\section{Instrumentos}

Se diseñó un instrumento para recoger datos sociodemográficos, que incluía las siguientes variables: diagnóstico oncológico, estadio de la enfermedad, tipo de tratamiento, sexo, edad, estado civil, cuidador primario, con quién vive el enfermo, lugar de residencia, nivel de estudios, situación laboral, afiliación religiosa y grado de participación religiosa (tabla 1).

El malestar emocional, la ansiedad y la depresión se evaluaron mediante la Escala de Ansiedad y Depresión Hospitalaria (HADS), en su versión en español. La validación colombiana mostró una adecuada consistencia interna y validez. El coeficiente alfa de Cronbach fue de .85 (Rico, Restrepo \& Molina, 2005). El HADS es el instrumento de referencia a la hora de evaluar depresión y ansiedad en población oncológica (Jacobsen et al., 2005; Carey, Noble, Sanson-Fisher \& Mackenzie, 2012). Consta de 14 ítems que evalúan dos subescalas, una de ansiedad y otra de depresión. Permite obtener un resultado global de malestar emocional (HADST), así como los específicos de ansiedad (HADSA) y depresión (HADSD). La intensidad o la frecuencia del síntoma se evalúa en una escala Likert de 4 puntos (rango 0-3). Los puntajes entre 0 y 7 son considerados normales; entre 8 y 10 , dudosos, y un puntaje mayor a 11, un problema clínico de depre- sión o ansiedad (Rico et al., 2005). Para el presente estudio se incluyeron las tres variables emocionales (malestar emocional global, ansiedad y depresión).

Tabla 1

Características demográficas de los pacientes

\begin{tabular}{|c|c|}
\hline Variable & $N(\%)$ \\
\hline Sexo femenino & $35(83.3)$ \\
\hline \multicolumn{2}{|l|}{ Estado civil } \\
\hline Soltero & $11(26.2)$ \\
\hline Divorciado/separado & $10(23.8)$ \\
\hline Casado/unión libre & $8(19.1)$ \\
\hline Viudo & $13(31.0)$ \\
\hline \multicolumn{2}{|l|}{ Cuidador primario } \\
\hline Cónyuge & $11(26.2)$ \\
\hline Hijo & $10(23.8)$ \\
\hline No tiene cuidador & $7(16.7)$ \\
\hline Otro familiar & $13(31.0)$ \\
\hline Otro & $1(2.4)$ \\
\hline \multicolumn{2}{|l|}{ Con quien vive } \\
\hline Con la familia & $40(95.2)$ \\
\hline Solo & $2(4.8)$ \\
\hline \multicolumn{2}{|l|}{ Lugar de residencia } \\
\hline Urbana & $38(90.5)$ \\
\hline Rural & $2(4.8)$ \\
\hline \multicolumn{2}{|l|}{ Nivel de estudios } \\
\hline Sin estudios & $0(0.0)$ \\
\hline Estudios primarios & $14(33.3)$ \\
\hline Estudios secundarios & $18(42.9)$ \\
\hline Estudios adicionales a los secundarios & $10(23.8)$ \\
\hline \multicolumn{2}{|l|}{ Situación laboral } \\
\hline Empleado & $10(23.8)$ \\
\hline Trabajador independiente & $5(11.9)$ \\
\hline Desempleado & $5(11.9)$ \\
\hline Jubilado/pensionado & $5(11.9)$ \\
\hline Ama de casa & $16(38.1)$ \\
\hline Otro & $1(2.4)$ \\
\hline
\end{tabular}




\begin{tabular}{lr}
\hline \multicolumn{1}{c}{ Variable } & $N(\%)$ \\
\hline \multicolumn{1}{c}{ Afiliación religiosa } & $39(92.9)$ \\
Católica & $2(4.8)$ \\
Cristiana & $1(2.4)$ \\
Evangélica & \\
$\quad$ Grado de participación en la religión & $3(7.1)$ \\
Creyente no participante & $39(92.9)$ \\
Creyente participante
\end{tabular}

La CP se evaluó mediante la Escala de Competencia Percibida (ECP), en su versión validada en población española (Fernández, Álvarez, Blasco, Doval \& Sanz, 1998). El instrumento está compuesto por 8 ítems que miden la expectativa que tienen los sujetos para manejar su entorno con eficacia, a través de una escala Likert de 6 puntos. El rango de puntuaciones totales fluctúa entre 8 y 48 puntos: a mayor puntuación hay una mayor competencia percibida. La media es de 35.4 y la desviación estándar es de 5.29; la consistencia interna, estimada a través del coeficiente alfa de Cronbach, es de .83 .

\section{Análisis estadístico}

Los datos se analizaron empleando el Paquete Estadístico para Ciencias Sociales (SPSS versión 19). Se obtuvieron estadísticos descriptivos para los datos sociodemográficos, médicos y clínicos. Se exploraron asociaciones entre las pruebas de estudio con variables de interés y se aplicaron las siguientes pruebas: (a) Kruskal-Wallis, para la diferencia de medianas de las pruebas por estadio y tratamiento recibido; (b) prueba de Fisher, para la diferencia de proporciones de pacientes según sexo y presencia de depresión, ansiedad y competencia percibida; (c) coeficiente de Pearson, para correlacionar los puntajes de las pruebas y la edad, y (d) alfa de Cronbach, para el análisis de la consistencia interna de las pruebas. En todos los casos, el resultado se consideró estadísticamente significativo cuando el valor de probabilidad era menor a .05 .

\section{Resultados}

Las siguientes son las características generales de los 42 enfermos que participaron: tenían una edad promedio de 49 \pm 10.6 años (rango 22-69), en su mayoría (83\%) fueron mujeres, y uno de cada tres eran viudos. El $26.2 \%$ eran cuidados por su pareja; el $90 \%$ provenía de zona urbana, el $67.7 \%$ tenía estudios secundarios y superiores, el $38.1 \%$ se desempeñaba como amas de casa, un $92.9 \%$ profesaba por religión la católica, e igual porcentaje eran creyentes participantes. En la tabla 1 se detalla la distribución de la muestra según sus datos demográficos. Como se aprecia en la tabla 2, el diagnóstico oncológico más frecuente fue cáncer de mama (26.2\%), el 61.9\% de los participantes se encontraba en estadio IV y más del $50 \%$ estaba en tratamiento paliativo.

Tabla 2

Características clínicas de los pacientes

\begin{tabular}{|c|c|}
\hline Variable & $N(\%)$ \\
\hline \multicolumn{2}{|c|}{ Diagnóstico oncológico } \\
\hline Mama & $11(26.2)$ \\
\hline Cuello uterino & $4(9.5)$ \\
\hline Colon & $4(9.5)$ \\
\hline Pulmón & $3(7.1)$ \\
\hline Recto & $3(7.1)$ \\
\hline Primario desconocido & $2(4.8)$ \\
\hline Gástrico & $2(4.8)$ \\
\hline Otros & $14(33.3)$ \\
\hline \multicolumn{2}{|l|}{ Estadio } \\
\hline I & $2(4.8)$ \\
\hline II & $4(9.5)$ \\
\hline III & $10(23.8)$ \\
\hline IV & $26(61.9)$ \\
\hline \multicolumn{2}{|c|}{ Tratamiento } \\
\hline Tratamiento activo & $17(40.5)$ \\
\hline Cuidados paliativos & $23(54.8)$ \\
\hline Libre de enfermedad & $2(4.8)$ \\
\hline
\end{tabular}


Con respecto a las variables de estudio, se encontró que en promedio los participantes puntuaron 7.8 en ansiedad (HADSA), 5.6 en depresión (HADSD) y 13.4 en malestar emocional (HADST), lo cual indica puntuaciones medias-bajas. La CP obtuvo una puntuación promedio de 37 , que muestra una CP moderada (tabla 3). En la tabla 4 se muestran los valores medianos y cuartiles 1 y 3 de

Tabla 3

Estadisticas descriptivas HADS y CP

\begin{tabular}{lrrrrrr}
\hline \multirow{1}{*}{ Pruebas } & Media & Desv. típ. & Mínimo & Máximo & \multicolumn{2}{c}{ Percentiles } \\
\hline Total ansiedad & 7.8 & 4.1 & 0.0 & 17.0 & 5.0 & 11.0 \\
Total depresión & 5.6 & 4.1 & 0.0 & 14.0 & 1.0 & 8.3 \\
HADS total & 13.4 & 7.5 & 0.0 & 31.0 & 7.0 & 19.0 \\
CP total & 37.0 & 7.7 & 20.0 & 48.0 & 33.8 & 42.3 \\
\hline
\end{tabular}

Tabla 4

Valores medianos y cuartiles 1 y 3 de las pruebas HADS total (HADST), HADS depresión (HADSD),

HADS ansiedad (HADSA) y CP según estadio y tratamiento

\begin{tabular}{|c|c|c|c|c|c|}
\hline Estadio & & HADSA & HADSD & HADST & $\mathrm{CP}$ \\
\hline & Cuartil 1 & .0 & .0 & .0 & 43.0 \\
\hline \multirow[t]{3}{*}{1} & Mediana & .0 & .0 & .0 & 43.0 \\
\hline & Cuartil 3 & .0 & .0 & .0 & 43.0 \\
\hline & Cuartil 1 & 1.3 & .3 & 2.0 & 39.3 \\
\hline \multirow[t]{3}{*}{2} & Mediana & 5.5 & 1.0 & 6.0 & 42.0 \\
\hline & Cuartil 3 & 6.8 & 8.5 & 15.3 & 45.5 \\
\hline & Cuartil 1 & 7.8 & 1.0 & 8.5 & 32.5 \\
\hline \multirow[t]{3}{*}{3} & Mediana & 10.0 & 6.5 & 16.0 & 39.5 \\
\hline & Cuartil 3 & 12.0 & 9.0 & 21.0 & 42.0 \\
\hline & Cuartil 1 & 4.8 & 2.8 & 8.5 & 32.5 \\
\hline \multirow[t]{4}{*}{4} & Mediana & 9.0 & 6.0 & 15.5 & 39.0 \\
\hline & Cuartil 3 & 11.0 & 8.0 & 19.0 & 42.3 \\
\hline & Chi-cuadrado de Kruskal-Wallis $(\mathrm{GL}=3)$ & 1.52 & 1.85 & 1.67 & 2.09 \\
\hline & Valor de $\mathrm{p}$ & .45 & .41 & .44 & .55 \\
\hline \multicolumn{6}{|l|}{ Tratamiento } \\
\hline & Cuartil 1 & 2.0 & 0.5 & 3.5 & 36.0 \\
\hline \multirow[t]{3}{*}{ Activo } & Mediana & 7.0 & 4.0 & 13.0 & 41.0 \\
\hline & Cuartil 3 & 11.0 & 9.5 & 19.5 & 43.5 \\
\hline & Cuartil 1 & 5.0 & 3.0 & 9.0 & 31.0 \\
\hline \multirow[t]{2}{*}{$\mathrm{CP}$} & Mediana & 9.0 & 6.0 & 16.0 & 39.0 \\
\hline & Cuartil 3 & 11.0 & 8.0 & 19.0 & 42.0 \\
\hline
\end{tabular}




\begin{tabular}{llrrrr}
\hline \multicolumn{1}{c}{ Estadio } & & HADSA & HADSD & HADST & CP \\
\hline \multirow{2}{*}{$\begin{array}{l}\text { Libre de la } \\
\text { enfermedad }\end{array}$} & Cuartil 1 & 8.0 & 8.0 & 16.0 & 20.0 \\
& Mediana & 10.0 & 8.5 & 18.5 & 29.0 \\
& Cuartil 3 & 2.0 & 0.5 & 3.5 & 36.0 \\
& Chi-cuadrado de Kruskal-Wallis $(G L=2)$ & 1.58 & 1.80 & 1.63 & 3.10 \\
& Valor de $p$ & 0.45 & 0.41 & 0.44 & 0.21 \\
\hline
\end{tabular}

las pruebas aplicadas según estadio y tratamiento. Es de resaltar que los índices de ansiedad, depresión y malestar emocional fueron más elevados en estadios más avanzados de la enfermedad, aunque no se encontró diferencia significante $(p<.05)$ de los puntajes de las pruebas por estadio, ni por tipo de tratamiento. Tampoco se encontraron diferencias estadísticamente significantes por sexo en las variables ansiedad y competencia percibida, a pesar que en depresión los hombres obtuvieron puntuaciones mucho más elevadas que las mujeres (tabla 5).

Tabla 5

Proporción de pacientes según sexo y presencia de depresión, ansiedad y competencia baja

\begin{tabular}{lccc}
\hline Variables & $\begin{array}{c}\text { Hombres } \\
(\%)\end{array}$ & $\begin{array}{c}\text { Mujeres } \\
(\%)\end{array}$ & $\begin{array}{c}\text { Valor de } p \\
\text { (prueba de } \\
\text { Fisher) }\end{array}$ \\
\hline Con depresión & 54.3 & 28.6 & .41 \\
Con ansiedad & 14.3 & 14.3 & 1.00 \\
Competencia baja & 28.6 & 28.6 & 1.00 \\
\hline
\end{tabular}

Se llevó a cabo un análisis correlacional entre las variables ansiedad, depresión, malestar emocional y CP. Se observó una relación inversa y significativa entre el malestar emocional y la CP $(p<.01)$; sin embargo, la fuerza de dicha relación fue de baja a moderada. No hubo correlación entre ansiedad o depresión y CP (tabla 6).

El análisis de la consistencia interna de las pruebas de estudio mostraron valores de alfa de Cronbach muy buenos: HADS: .85 y CP: .81.
Tabla 6

Correlaciones de Pearson entre las variables edad, pruebas HADS total (HADST), HADS depresión (HADSD), HADS ansiedad (HADSA) y CP(CP)

\begin{tabular}{lccccc}
\hline & Edad & HADSA & HADSD & HADST & CP \\
\hline Edad & 1.00 & & & & \\
HADSA & $-.42^{* *}$ & 1.00 & & & \\
HADSD & -.21 & $.67^{* *}$ & 1.00 & & \\
HADST & $-.35^{*}$ & $.92^{* *}$ & $.91^{* *}$ & 1.00 & \\
CP & .16 & -.28 & -.28 & $-.31^{*}$ & 1.00 \\
\hline
\end{tabular}

* La correlación es significante al nivel .05 (bilateral).

${ }^{* *}$ La correlación es significativa al nivel .01 (bilateral).

\section{Discusión}

Respecto a las características de la población, se evidencia un porcentaje mayor de población femenina con prevalencia de diagnósticos de cáncer de mama y cuello uterino. Dichos resultados coinciden con los datos aportados por el Instituto Nacional de Cancerología para el periodo 2002-2006 (2010), cuando estos diagnósticos fueron los más prevalentes tanto de la región de Antioquia como para Colombia. Adicionalmente, concuerdan con prevalencias de mortalidad por cáncer registradas por la Dirección Seccional de Salud de Antioquia (2012), donde, después del cáncer de pulmón y próstata, las más altas son debidas a cáncer de mama y cuello uterino, datos relevantes si se tiene en cuenta el avanzado estadio de la enfermedad en un elevado porcentaje de la muestra. El $61.9 \%$ de la población de este estudio se encontraba en un estadio IV de la enfermedad, y un $54.8 \%$, en tratamiento con cuidados paliativos. 
Como se comentó, la enfermedad en estado avanzado trae múltiples consecuencias físicas y cambios psicológicos, sociales y espirituales que requieren el empleo de estrategias para lograr la adaptación (Krikorian, Limonero \& Maté, 2012). Dichas condiciones hacen más difícil el ajuste, particularmente cuando el diagnóstico inicial se ha hecho en un estadio ya avanzado, como es frecuente encontrar en nuestra población.

Teniendo en cuenta lo anterior, así como el elevado porcentaje de pacientes con enfermedad avanzada que participaron en el estudio, no es de extrañar que más de la mitad de los hombres y cerca del $30 \%$ de las mujeres hayan presentado síntomas de depresión clínicamente significativos; mientras que alrededor del $15 \%$ de los participantes tuvo síntomas de ansiedad clínicamente relevantes. Sin embargo, las puntuaciones medias obtenidas en ansiedad, depresión y malestar emocional fueron medias-bajas, según lo evaluado a través del HADS y empleando los puntos de corte recomendados para la población.

Los índices de depresión en las mujeres fueron similares a los datos encontrados en el estudio de Ornelas-Mejorada et al. (2011), mientras que los de depresión en hombres fueron más elevados, incluso que aquellos encontrados en el estudio de Delgado-Guay et al. (2009). La prevalencia de depresión en pacientes con cáncer avanzado varía de forma importante con cifras que van desde el 5 al 26\% (Irving \& Lloyd-Williams, 2010), dependiendo de los criterios que se empleen para evaluarla, mientras que entre el 15 y el $50 \%$ de los pacientes con cáncer pueden presentar síntomas depresivos a lo largo de la trayectoria de la enfermedad (Rosenstein, 2011). En una amplia revisión de la literatura llevada a cabo por Teunissen et al. (2007), se encontró que los síntomas de ansiedad y depresión tuvieron prevalencias del 48 y $39 \%$, respectivamente.

Si bien los índices de depresión coinciden con los reportes de la literatura, en el presente estudio los niveles de ansiedad reportados por los pacien- tes fueron menores que aquellos encontrados en diversos estudios (Delgado-Guay et al., 2009; Ornelas-Mejorada et al., 2011). De acuerdo con Miovic y Block (2007), los síntomas de ansiedad clínicamente relevantes ocurren entre el 25 y $48 \%$ de los pacientes con cáncer; mientras que entre el 2 y $14 \%$ de aquellos con enfermedad avanzada cumplen criterios para un trastorno de ansiedad.

Los pacientes con enfermedades avanzadas y terminales presentan más limitaciones en sus actividades diarias y son más dependientes, debido a su condición de discapacidad, que afecta la percepción de control sobre la situación y su desempeño, a la vez que esto impacta en su estado emocional (Bárez et al., 2003). Por ejemplo, un estudio japonés sobre la relación entre la autoeficacia, el ajuste psicológico y las condiciones físicas en pacientes con cáncer avanzado sugiere que los pacientes con mejor estado físico mostraban mejor autoeficacia y menor angustia emocional. Ello indicao que las condiciones de salud tienen un impacto importante en la adaptación de los pacientes a la enfermedad y a los tratamientos (Hirai et al., 2002).

Los resultados del presente estudio señalan una relación inversa y significativa entre el malestar emocional y la CP. Es decir, mientras mayor fue el malestar emocional manifestado por los pacientes, menores fueron sus niveles de CP. Estos resultados encuentran sustento en la propuesta teórica de Thompson (1981), según la cual las creencias de control influyen en la adaptación y promueven acciones directas para alterar las situaciones que resultan negativas para los sujetos $\mathrm{y}$, a la vez, facilitar la respuesta emocional.

La sensación de control, el reconocimiento de habilidades propias y la expectativa de poder lograr lo que se propone posibilitan que los pacientes tomen una actitud activa frente a su proceso. En este sentido, la expectativa de control, sumada a la expectativa de resultado (constructo conocido como CP), puede facilitarles a los pacientes el paso de ser agentes pasivos a asumir un rol activo y sentirse útiles en el medio en el que están inmersos. 
En particular, dicho constructo explicaría cómo los pacientes con cáncer, a pesar de estar expuestos a situaciones que tienen un significado con un gran peso emocional, (por ejemplo, enfrentar la posibilidad de muerte), logran reducir las consecuencias negativas del estrés, promoviendo un modo eficaz de afrontamiento ante la situación. Esto es de especial relevancia en pacientes como los que participaron en esta muestra, en que en su mayoría se encontraban en estadios avanzados y bajo tratamiento paliativo.

Neipp, López-Roig, Terol y Pastor (2009), en su estudio sobre cambios en las creencias de control, estado emocional y adaptación psicosocial en mujeres con cáncer, encontraron que el mejor predictor del estado emocional fue la creencia de $\mathrm{CP}$ en salud; asimismo, las creencias generales y específicas de control tuvieron capacidad predictiva en la adaptación. En otro estudio llevado a cabo por Mystakidou et al. (2010), se halló una correlación negativa entre autoeficacia (creencia específica de control) y depresión en mujeres y hombres con cáncer, lo que aporta evidencia adicional a la relación entre el estado emocional y las creencias de control. Un estudio adicional llevado a cabo por el mismo grupo de investigadores, acerca de autoeficacia y ansiedad en pacientes crónicos y con cáncer atendidos en una unidad de cuidados paliativos, obtuvo como resultado una correlación negativa significativa entre ansiedad y autoeficacia (Mystakidou et al., 2012). En conjunto, dichos resultados aportan evidencia a la relación entre las creencias de control, sean estas específicas o generales, y el malestar emocional y la adaptación. En ese sentido, respaldan algunos resultados obtenidos en nuestro estudio, ya que las creencias generales de control (CP) correlacionaron de manera inversa con el malestar emocional, a pesar de que no lo hicieron de forma específica con la ansiedad. Sería interesante que futuros estudios exploraran la relación diferencial entre las creencias de control específicas y generales y el malestar emocional global y los estados más específicos de ansiedad y depresión.
De tener en cuenta lo anterior, es importante mencionar algunas limitaciones del presente estudio. En primer lugar, se realizó un muestreo por conveniencia y se incluyeron en el estudio pacientes que asistían a la consulta psicológica por primera vez. Es posible que ello introduzca sesgos de selección y que los índices de malestar emocional y CP sean diferentes en la población que no consulta al servicio de psicología. En futuros estudios sería importante hacer un muestreo aleatorio que incluya pacientes de la consulta general oncológica. En segundo lugar, el tamaño muestral fue relativamente bajo, lo cual puede limitar la detección de relaciones que en otro caso podrían haber sido significativas o más fuertes (por ejemplo, la relación entre ansiedad, depresión y competencia percibida). Finalmente, sería importante incluir en futuras investigaciones variables que evalúen el grado de adaptación, más allá del estado emocional, así como la relación entre la CP y los estilos o estrategias de adaptación de los pacientes.

\section{Conclusiones}

En conjunto, si bien la investigación sobre $\mathrm{CP}$ en cáncer es aún escasa, los estudios indican que tanto las creencias de control generales como las específicas mantienen una relación estrecha con el malestar emocional y la adaptación. El presente estudio evidenció la relación existente entre la CP (una creencia global de control) y el malestar emocional en una muestra de pacientes con cáncer. El investigar sobre la CP tiene ventajas importantes, especialmente cuando los pacientes enfrentan situaciones de enfermedad oncológica, la cual puede ser sumamente variable e impredecible, y donde las creencias generales de control favorecen la adaptación a diversos panoramas a lo largo del trayecto de enfermedad. En este sentido, los resultados del presente estudio tienen importantes implicaciones en la psicología clínica en el contexto oncológico, puesto que pone de relieve la posibilidad de im- 
pactar el bienestar emocional, al hacer hincapié en el fortalecimiento de la competencia percibida de los pacientes, incluso en etapas avanzadas de la enfermedad.

\section{Referencias}

Abraído-Lanza, A. F. (1997). Latinas with arthritis: Effects of illness, role identity, and competence on psychological well-being. American Journal of Community Psychology, 25(5), 601-627.

Aubin, G., Hachey, R. \& Mercier, C. (1999). Meaning of daily activities and subjective quality of life in people with severe mental illness. Scandinavian Journal of Occupational Therapy, 6(2), 53-62.

Bandura, A. (1986). Social foundations of thought and action: A social cognitive theory. Englewood Cliffs, NJ: Prentice-Hall.

Bárez, M., Blasco, T. \& Fernández, J. (2003). La inducción de sensación de control como elemento fundamental de la eficacia de las terapias psicológicas en pacientes de cáncer. Anales de Psicología, 19(2), 235-246.

Bultz, B. \& Johansen, C. (2011). Screening for distress, the 6th vital sing: Where are we, and where are we going? Psychooncology, 20, 269-571.

Bultz, B. \& Carlson, L. (2006). Emotional distress: The six vital sing-future directions in cancer care. Psychooncology, 15, 93-95.

Carey, M., Noble, N., Sanson-Fisher, R. \& Mackenzie, L. (2012). Identifying psychological morbidity among people whit cancer using the hospital Anxiety and Depression Scale: Time to revisit first principles? Psychooncology, 21(3), 229-238.

Carlson, L. E., Angen, M., Cullum, J., Goodey, E., Koopmans, J., Lamont, L... Bultz, B. D. (2004). High levels of untreated distress and fatigue in cancer patients. British Journal of Cancer, 90(12), 2297-2304.

Costa, G. \& Ballester, R. (2010). Influencia de las características sociodemográficas y clínicas en la calidad de vida y malestar emocional del paciente oncológico. Psicooncología, 7(2-3), 453-462.

Cunningham, A. J., Lockwood, G. A. \& Cunningham, J. A. (1991). A relationship between perceived self-efficacy and quality-of-life in cancer-patients. Patient Education and Counseling, 17(1), 71-78.

Delgado-Guay, M., Parsons, H. A., Li, Z., Palmer, J. L. \& Bruera, E. (2009). Symptom distress in advanced cancer patients with anxiety and depression in the palliative care setting. Supportive Care in Cancer, 17(5), 573-579. doi: 10.1007/ s00520-008-0529-7

Dirección Seccional de Salud de Antioquia. (2012). Registro poblacional de cáncer de antioquia. DSSA: datos preliminares 2007-2009. Recuperado de: http://www.dssa.gov.co

Fernández, C., Villoria, E., Amigo, I., Padierna, C., Fernández, R. \& Peláez, I. (2012). Influencia del estado emocional en la sintomatología referida por paciente con cáncer de mama de pulmón durante el tratamiento con quimioterapia. $\mathrm{Me}$ dicina Paliativa, 20(3), 85-92.

Fernández, J. \& Edo, S. (1994). ¿Cómo influye el control percibido en el impacto que tienen las emociones sobre la salud? Anales de Psicología, 10(2), 127-133.

Fernández, J., Álvarez, M., Blasco, T., Doval, E. \& Sanz, A. (1998). Validación de la escala de competencia personal de Wallston: implicaciones para el estudio del estrés. Revista de Ansiedad y Estrés, 4(1), 31-41.

Finck, C. \& Forero, M. J. (2011). Ansiedad y depresión en pacientes con cáncer de seno y su relación con la espiritualidad/religiosidad. $R e$ vista de Psicología Universidad de Antioquia, 3(1), 7-27.

Hernández, M., Cruzado, J. A., Prado, M. C., Rodríguez, E., Hernández, C., González, M.A... Martín, J. C. (2012). Salud mental y malestar emocional en pacientes con cáncer. Psicooncología, 9(2-3), 233-257. 
Hirai, K., Suzuki, Y., Tsuneto, S., Ikenaga, M., Hosaka, T. \& Kashiwagi, T. (2002). A structural model of the relationships among self-efficacy, psychological adjustment, and physical condition in Japanese advanced cancer patients. Psychooncology, 11(3), 221-229.

Instituto Nacional de Cancerología. (2010). Plan nacional para el control del cáncer en Colombia 2010-2019. Bogotá: Ministerio de la Protección Social, República de Colombia.

International Agency for the Research of Cancer. (2014). World Cancer Report 2014. Press release $n^{\circ} 224$. London: WHO. Recuperado de http://www.iarc.fr/en/media-centre/pr/2014/ pdfs/pr224_E.pdf

Irving, G. \& Lloyd-Williams, M. (2010). Depression in advanced cancer. European Journal of Oncology Nursing, 14(5), 395-399. doi: 10.1016/j. ejon.2010.01.026

Jacobsen, P. B., Donovan, K. A., Trask, P. C., Fleishman, S. B., Zabora, J., Baker, F.... Holland, J. C. (2005). Screening for psychologic distress in ambulatory cancer patients. Cancer, 103(7), 1494-1502.

Kelly, B., McClement, S. \& Chochinov, H. M. (2006). Measurement of psychological distress in palliative care. Palliative Medicine, 20(8), 779-789.

Krikorian, A. (2008). Valoración del sufrimiento en cáncer avanzado. Psicooncología, 5(2-3), 257-264.

Krikorian, A., Limonero, J. T. \& Maté, J. (2012). Suffering and distress at the end-of-life. Psychooncology, 21(8), 799-808. doi: 10.1002/pon.2087

Limonero, J. (1996). El fenómeno de la muerte en la investigación de las emociones. Revista de Psicología Generaly Aplicada, 49(2), 249-265.

Limonero, J., Tomás-Sábado, J., Fernández-Castro, J., Cabellas, R. \& Gómez, J. (2010). Competencia personal percibida y ansiedad ante la muerte en estudiantes de enfermería. Revista de Ansiedad y Estrés, 16(2-3), 177-188.

Martín, M., Pastor, M. A., Lledó, A., López-Roig, S., Terol, M. C. \& Rodríguez, J. (2001). Percep- ción de control en el síndrome fibromiálgico. Psicothema,13(4), 586-591.

Ministerio de Salud y Protección Social, Instituto Nacional de Cancerología. (2012). Plan decenal para el control del cáncer en Colombia 20122021. Bogotá: Ministerio de Salud y Protección Social de Colombia.

Miovic, M. \& Block, S. (2007). Psychiatric disorders in advanced cancer. Cancer, 110(8), 1665-1676.

Mitchell, A. J., Karr, S., Coggan, C. \& Herdman, J. (2008). Acceptability of common screening methods used to detect distress and related mood disorders-preferences of cancer specialists and non-specialists. Psychoonchology,17(3), 226236.

Mystakidou, K., Parpa, E., Tsilika, E., Gogou, P., Panagiotou, I., Galanos, A... Gouliamos, A. (2010). Self-efficacy, depression, and physical distress in males and females with cancer. American Journal of Hospice and Palliative Care, 27(8), 518-525. doi: 10.1177/1049909110376808

Mystakidou, K., Tsilika, E., Parpa, E., Panagiotou, I., Galanos, A. \& Gouliamos, A. (2012). Differences in levels of self-efficacy and anxiety between cancer and chronically-ill patients attending a Palliative Care Unit. Journal of BUON, 17(4), 785-790.

Neipp, M.C., López-Roig, S., Terol, M.C. \& Pastor, M.A. (2009). Cambios en las creencias de control, status emocional y adaptación psicosocial en mujeres con cáncer de mama. Anales de Psicología, 25(1), 36-43.

Ornelas-Mejorada, R., Tufiño, M. \& Sánchez- Sosa, J. J. (2011). Ansiedad y depresión en mujeres con cáncer de mama en radioterapia: prevalencia y factores asociados. Acta de Investigación Psicológica, 1(3), 401-414.

Porter, L. S., Keefe, F. J., Garst, J., McBride, C. M. \& Baucom, D. (2008). Self-efficacy for managing pain, symptoms, and function in patients with lung cancer and their informal caregivers: associations with symptoms and distress. Pain, 137(2), 306-315. 
Rebollo, M., \& Aguilar, S. (2006). Distrés y cáncer. Revista Neurología y Psiquiatría, 39(1), 52-58.

Rico, J., Restrepo, M. \& Molina, M. (2005). Adaptación y validación de la escala Hospitalaria de Ansiedad y Depresión (HAD) en una muestra de pacientes con cáncer del Instituto Nacional de Cancerología de Colombia. Avances en Medición, 3, 73-86.

Rosenstein, D. L. (2011). Depression and end-of-life care for patients with cancer. Dialogues in Clinical Neuroscience, 13(1), 101-108.

Rueda, B. \& Pérez-García, A. M (2004). Análisis comparativo de la CP general y la específica de salud. Revista Ansiedad y Estrés, 10(1), 127-139.

Rueda, B. \& Pérez-García, A. M. (2004). Personalidad y percepción de autoeficacia: influencia sobre el bienestar y el afrontamiento de los problemas de salud. Revista de Psicopatología y Psicología Clínica, 9(3), 205-219.

Rueda, B., Pérez-García, A. \& Bermúdez, J. (2003). La salud emocional dese la perspectiva de la competencia percibida. Acción Psicológica, 2(1), 41-49.

Smith, N., Kielhofner, G. \& Watts, J. (1986). The relationships between volition, activity pattern, and life satisfaction in the elderly. American Journal of Occupational Therapy, 40(4), 278-283.

Teunissen, S. C., Wesker, W., Kruitwagen, C., de Haes, H. C., Voest, E. E. \& de Graeff, A. (2007). Symptom prevalence in patients with incurable cancer: a systematic review. Journal of Pain and Symptom Management, 34(1), 94-104.

Thompson, S. C. (1981). Will it hurt less if i can control it?: A complex answer to a simple question. Psychological Bulletin, 90(1), 89-101.

Williams, G. C., Niemiec, C. P., Patrick, H., Ryan, R. M. \& Deci, E. L. (2009). The importance of supporting autonomy and perceived competence in facilitating long-term tobacco abstinence. Annals of Behavioral Medicine, 37(3), 315-324. doi: 10.1007/s12160-009-9090-y

World Health Organization. (2008). World cancer report 2008. Geneve: World Health Organization.

\section{Fecha de recepción: 24 de mayo de 2014} Fecha de aceptación: 15 de abril de 2015 